Document downloaded from:

http://hdl.handle.net/10251/52834

This paper must be cited as:

March Iborra, MDC.; García Narbón, JV.; Sánchez, A.; Arnau Vives, A.; Jiménez Jiménez, Y.; García, P.; Manclus Ciscar, JJ.... (2015). High-frequency phase shift measurement greatly enhances the sensitivity of QCM immunosensors. Biosensors and Bioelectronics. (65):1-8. doi:10.1016/j.bios.2014.10.001.

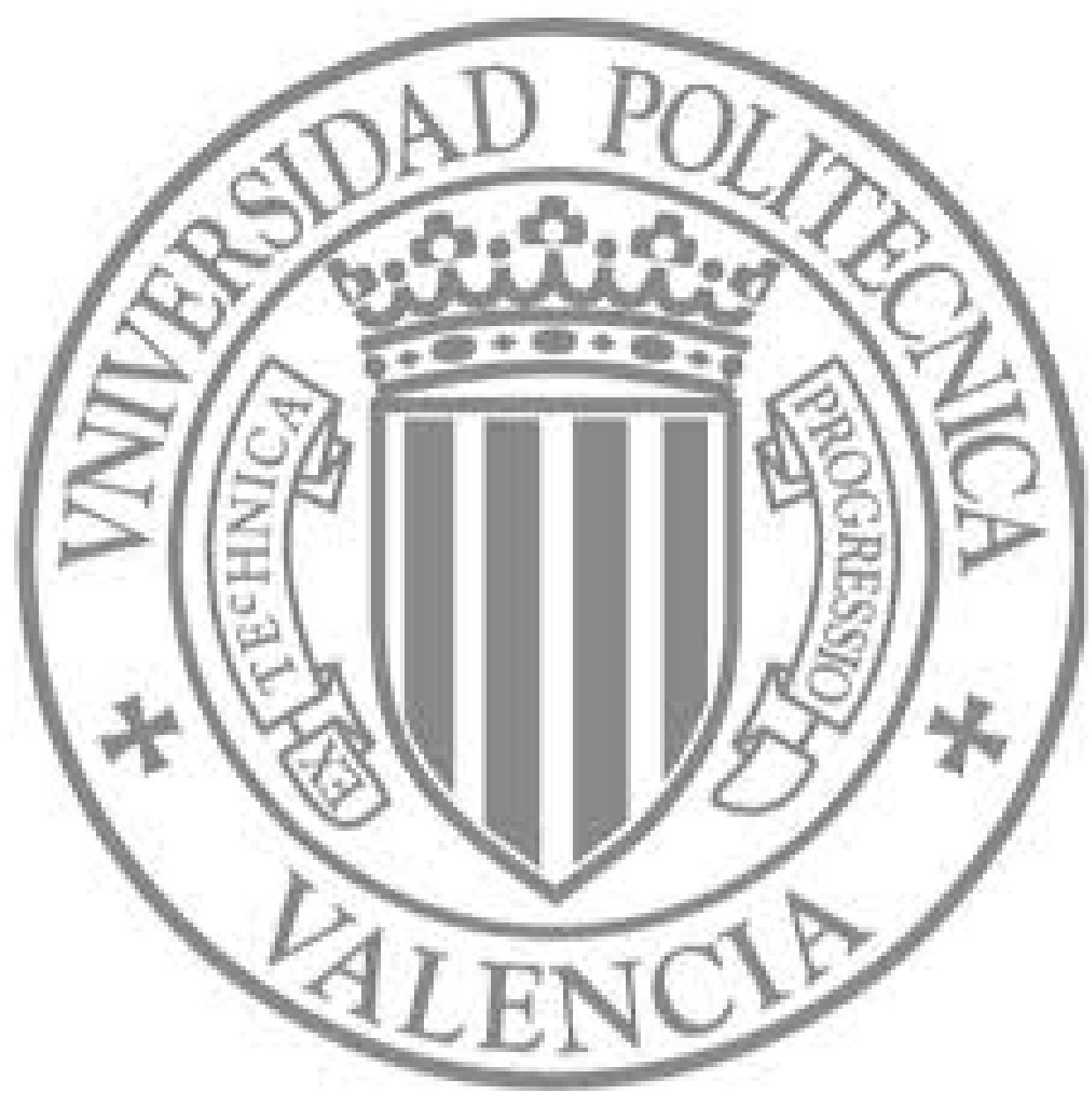

The final publication is available at

http://dx.doi.org/10.1016/j.bios.2014.10.001

Copyright Elsevier 


\section{High-Frequency phase shift measurement greatly enhances the sensitivity of QCM immunosensors}

Carmen March $^{\mathrm{a}, \mathrm{b}, *}$, José V. García ${ }^{\mathrm{b}, \mathrm{c}}$, Ángel Sánchez ${ }^{\mathrm{a}}$, Antonio Arnau ${ }^{\mathrm{b}, \mathrm{c}}$, Yolanda Jiménez $^{\mathrm{b}, \mathrm{c}}$, Pablo García ${ }^{\mathrm{b}}$, Juan J. Manclús ${ }^{\mathrm{a}}$, Ángel Montoya ${ }^{\mathrm{a}}$

${ }^{a}$ Instituto Interuniversitario de Investigación en Bioingeniería y Tecnología Orientada al Ser Humano (I3BH), Universitat Politècnica de València, Camino de Vera s/n, Edificio $8 B-N$, 46022 Valencia, Spain.

${ }^{b}$ Advanced Wave Sensors S.L. Valencia, Spain.

${ }^{c}$ Grupo de Fenómenos Ondulatorios (GFO). Universitat Politècnica de València, Camino de Vera s/n, Edificio 7F, 46022 Valencia, Spain.

*Corresponding author. Tel and fax: +34 963877093.

E-mail address: cmarch@ginmuno.i3bh.es 


\section{ABSTRACT}

In spite of being widely used for in liquid biosensing applications, sensitivity improvement of conventional (5-20 MHz) quartz crystal microbalance (QCM) sensors remains an unsolved challenging task. With the help of a new electronic characterization approach based on phase change measurements at a constant fixed frequency, a highly sensitive and versatile high fundamental frequency (HFF) QCM immunosensor has successfully been developed and tested for its use in pesticide (carbaryl and thiabendazole) analysis. The analytical performance of several immunosensors was compared in competitive immunoassays taking carbaryl insecticide as the model analyte. The highest sensitivity was exhibited by the $100 \mathrm{MHz}$ HFF-QCM carbaryl immunosensor. When results were compared with those reported for $9 \mathrm{MHz}$ QCM, analytical parameters clearly showed an improvement of one order of magnitude for sensitivity (estimated as the $\mathrm{I}_{50}$ value) and two orders of magnitude for the limit of detection (LOD): $30 \mu \mathrm{g} \mathrm{L}^{-1}$ vs $0.66 \mu \mathrm{g} \mathrm{L}^{-1} \mathrm{I}_{50}$ value and $11 \mu \mathrm{g} \mathrm{L}^{-1}$ vs $0.14 \mu \mathrm{g} \mathrm{L}^{-1} \mathrm{LOD}$, for 9 and $100 \mathrm{MHz}$, respectively. For the fungicide thiabendazole, $\mathrm{I}_{50}$ value was roughly the same as that previously reported for SPR under the same biochemical conditions, whereas LOD improved by a factor of 2 . The analytical performance achieved by high frequency QCM immunosensors surpassed those of conventional QCM and SPR, closely approaching the most sensitive ELISAs. The developed $100 \mathrm{MHz}$ QCM immunosensor strongly improves sensitivity in biosensing, and therefore can be considered as a very promising new analytical tool for in liquid applications where highly sensitive detection is required.

Key Words: High Fundamental Frequency; HFF QCM; Immunosensor; Sensitivity enhancement. 


\section{Introduction}

Throughout the last two decades, quartz crystal microbalance (QCM) resonators have gained an increasing relevance for biological and biochemical research. Since acoustic QCM sensors can detect mass and viscosity alterations on its surface by means of variations on their resonance frequency (Marx, 2003), they have found widespread acceptance as valuable tools for biomolecular interactions and related phenomena analysis. Their scientific merits, simple operation principles and affordable basic elements have been extensively discussed (Marx, 2007; Sagmeister et al., 2009; Speight and Cooper, 2012).

Already established and commercially available systems usually employ frequencies ranging from 5 to $20 \mathrm{MHz}$ (Stehrer et al., 2010; Uttenthaler et al., 2001; Yoshimine et al., 2011). According to Sauerbrey's relation (Sauerbrey, 1959), since mass sensitivity is directly proportional to the square of its resonance frequency $f_{0}$, the performance of a QCM sensor depends crucially on its fundamental resonance frequency. This relationship is expressed as follows:

$$
\Delta f=-2 f_{0}^{2} / \sqrt{\rho \mu} \cdot \Delta m
$$

where $\Delta f$ is the frequency shift produced as a consequence of a mass alteration per unit active area $\Delta m ; f_{0}$ is the fundamental resonance frequency with no attached mass; $\rho$ the quartz density (2648 $\left.\mathrm{g} \mathrm{cm}^{-3}\right)$; and $\mu$ the quartz shear modulus $\left(29.47 \mathrm{dyn}^{-2}\right.$, for ATcut).

Operation at suitable high fundamental frequencies (HFF) turns to be imperative for in liquid applications in which the sensitive detection of small changes is required. Uttenthaler and coworkers first developed a HFF-QCM biosensor for phage detection in liquids (Uttenthaler et al., 2001), but very few research groups have been working with HFF resonators in liquid media since this first application was demonstrated. HFF resonators are usually described as small, fragile and difficult to implement in flow-cell systems (Lin et al., 1993; Sagmeister et al., 2009; Stehrer et al., 2010). Moreover, these sensors do not quite get a stable resonance frequency signal in the presence of even minute fluctuations of hydrostatic pressure (Sota et al., 2002).

The fabrication of HFF sensors involves a reduction of their plate thickness. The thinned resonator region needs to be essentially carved into a thicker quartz plate otherwise mechanical stability and easy handling and installation in a flow-cell system 
would be restricted. These requirements, together with those derived from working in liquid media, are accomplished by a suitable "inverted mesa" design. This way, the thickness is achieved by chemically etching of only a small sensor central circular area. Likewise, the surrounding thicker material provides better stability whereas the sensitive central area could be as thinner as $8.3 \mu \mathrm{m}$ for up to $200 \mathrm{MHz}$ high frequencies (Michalzik et al., 2005; Sagmeister et al., 2009).

As mentioned above, higher sensitivity can be achieved when frequency increases. However, in oscillators mainly used with conventional low fundamental frequency resonators, unsteady frequency signal is largely caused because phase noise increases with higher frequencies. This way, resonance signal becomes unstable and therefore the pursued improvement remains unsolved. In order to reduce this noise, a new electronic characterization system has recently been proposed by our group (Montagut et al., 2011), based on the phase/mass relationship at a fixed frequency:

$$
\Delta \varphi \approx-\left(\Delta m / m_{L}\right)
$$

where $\Delta \varphi$ is the phase shift produced by a mass alteration $\Delta m$ on the sensor surface; and $\mathrm{m}_{\mathrm{L}}$ is the liquid mass perturbed by the resonator. When fundamental resonance frequency increases because crystal thickness decreases, $\Delta \varphi$ rises as $m_{L}$ is reduced. Therefore, if frequency is fixed and changes in phase are measured, operation at high frequencies without a significant increase of noise would be allowed and suitable phase shift $(\Delta \varphi)$ responses will be obtained.

Affinity interactions are often real-time monitored by means of transducer systems which allow label-free detection of the binding reaction. Although the most popular are optical systems based on surface plasmon resonance (SPR), the application of mass sensitive transducers as HFF QCM could turn this acoustic technology into a competitive tool for sensitivity improvement. In a previous work we described a piezoelectric immunosensor for pesticide determination (March et al., 2009), based on a conventional $9 \mathrm{MHz}$ QCM sensor. The achieved immunosensor sensitivity was enough for pesticide determination in fruit juice samples. However, it still was far from that required by more demanding applications, such as the measurement of pesticide residues at European regulatory levels in drinking water.

It is well know that enhanced sensitivity in competitive immunoassays for low molecular weight compounds can be achieved by lowering immunoreagent concentrations. The signal produced in biomolecular interactions must be, meantime, high enough even at low immunoreagent and analyte concentrations. Taking advantage 
of QCM measurements based on the phase/mass approach above described, in the present work we report the development of an HFF-QCM immunosensor for the highsensitivity determination of carbaryl and thiabendazole pesticides. Carbaryl, the first successful carbamate insecticide with a broad-spectrum efficacy, was chosen as a model analyte because it has become a reference analyte for immunoassay comparison, from ELISA to immunosensors (Abad et al., 1997; March et al., 2009; Mauriz et al., 2006). Thiabendazole (TBZ) is a widespread fungicide used in postharvest control of fungal diseases on fruit and vegetables. Moreover, it is one of the most widely detected pesticides on Europe and USA. The structures of both analytes are provided as supplementary information, Fig. S1.

\section{Material and Methods}

\subsection{Chemicals and immunoreagents}

Bovine serum albumin (BSA) fraction V was purchased from Roche Diagnostics (Mannheim, Germany). Tween-20 was supplied by Fluka-Aldrich Chemie (Buchs, Switzerland). All other chemicals were of analytical grade.

Reagents for covalent immobilization: 1-ethyl-3 (-3 dimethyl-amino-propyl) carbodiimide hydrochloride (EDC) and N-hydroxysuccinimide (NHS) were purchased from Pierce (Rockford, IL); thioctic acid (TA), mercaptohexadecanoic acid (MHDA) and 11-mercaptoundecanol (MUD) were supplied by Sigma-Aldrich Chemie (Steinheim, Germany); ethanolamine blocking agent was obtained from Sigma (St. Louis, MO).

Analytical pesticide standards: Carbaryl was purchased from Dr. Ehrenstorfer (Augsburg, Germany) and TBZ from Riedel-de Häen (Seelze, Germany).

Immunoreagents: For carbaryl assay, BSA-CNH protein-hapten conjugate and LIBCNH45 monoclonal antibody (MAb) were produced as previously described (Abad et al., 1997). For TBZ assay, BSA-TN3C protein-hapten conjugate and LIB-TN3C13 MAb were previously prepared as described (Abad et al., 2001). 


\subsection{HFF-QCM sensor set-up}

\subsubsection{HFF-QCM sensor chip and flow-cell assembly}

Sensors were from AWSensors (Valencia, Spain, www.awsensors.com). They were based on square shaped 50 and $100 \mathrm{MHz}$ AT-cut, inverted mesa crystals, with 36 $\mathrm{mm}^{2}$ of total area and an etched area thickness of approximately 33 and $17 \mu \mathrm{m}$, for 50 and $100 \mathrm{MHz}$, respectively. Electrode active surface ranged from 0.196 to $3.142 \mathrm{~mm}^{2}$ (for 0.5 to $2.0 \mathrm{~mm}$ electrode diameter). Sensors were assembled on a polyether ether ketone (PEEK) support with a conical hole to expose the active surface of the gold electrode where interface events as immunoassays will take place (supplementary information, Fig S2-A).

HFF-QCM chips were subsequently mounted into a flow-cell suitable for operation at high frequencies. The flow-cell was made up of two main components: the upper and the lower cell housing. Sensor chips were placed on the aluminum lower housing. Thereafter, they were sandwiched between this lower piece and a polymethyl methacrylate (PMMA) upper lid. The upper and lower pieces were held together by means of two screws making a $2.75 \mu$ internal volume flow-through cell chamber that was sealed either with a Polydimethylsyloxane (PDMS) or a nitrile O-ring. This way, only one face of the sensor was allowed to be in contact with the fluid (supplementary information, Fig S2-B).

\subsubsection{Measurement platform}

The flow cell was included in the AWS A10 test platform from AWSensors. This platform consists basically on an automated flow-through equipment controlled by syringe pumps (Hamilton, Bonaduz, GR, Switzerland) and thermostatized at $25^{\circ} \mathrm{C}$ combined with an electronic characterization system based on the fixed frequency phase-shift measurement technique described elsewhere (Montagut et al., 2011) (supplementary information, Fig S2-C). By means of this platform, before and after covalent functionalization, HFF-sensor chips were electronically characterized by impedance analysis performed in working buffer to determine the optimum operation frequency. In this work the chosen frequency was that at which the sensor showed the maximum conductance.

AWS A10 platform allows the remote control of fluidics, as well as data acquisition and analysis by AW-BIO v1.8 dedicated software (AWSensors, Spain). 
Phase, amplitude and temperature were therefore measured, recorded and processed through the assays. Samples were delivered to the flow cell by means of the syringe pump at a constant flow rate. Teflon tubing, internal diameter 0.25 and $0.80 \mathrm{~mm}$ were used for flow-through in and out port connections, respectively (Valco Vici, Switzerland and Supelco, Bellefonte, PA).

\subsection{Gold electrode surface functionalization}

\subsubsection{Clean up of crystals.}

50 and $100 \mathrm{MHz}$ HFF-QCM crystals assembled in the PEEK support were subjected to UV-ozone radiation for 15-20 min using a UV/Ozone ProCleaner from BioForce Nanosciences (Ames, IA). After the cleaning treatment, crystals were rinsed with distilled water and ethanol and blown dry with a stream of nitrogen gas.

\subsubsection{Covalent immobilization.}

Surface functionalization of HFF-QCM sensors was based on the classical amide chemistry to obtain self-assembled monolayers (SAM). All procedures were performed on only one face of the crystal following the previously described protocol (March et al., 2009), with the exception of:

- For carbaryl assays, the thiolated compounds used for SAM formation were TA or MHDA at concentrations ranging from $5 \mu \mathrm{M}$ to $10 \mathrm{mM}$ for BSA-CNH conjugate immobilization. For TBZ, a $250 \mu \mathrm{M}$ solution of MUD and MHDA (50:1 molar ratio) was used for BSA-TN3C conjugate immobilization.

- BSA-hapten conjugates in 0.1 M sodium phosphate buffer, $\mathrm{pH} 7.5$ was placed on the crystal active surfaces at concentrations ranging from $1.0 \mu \mathrm{g} \mathrm{mL}^{-1}$ to $10.0 \mathrm{mg} \mathrm{mL}^{-1}$ (carbaryl) or $20.0 \mu \mathrm{g} \mathrm{mL}^{-1}$ (TBZ), for $4 \mathrm{~h}$.

Functionalized sensors could be used for at least 75 times.

\subsection{Immunoassay format}

The indirect competitive immunoassays developed to determine carbaryl and TBZ were binding inhibition tests based on the conjugate-coated format. For the inhibition assays, a fixed amount of the respective monoclonal antibody was mixed with standard solutions of the analyte, and the mixture was pumped over the sensor surface 
previously immobilized with the assay conjugate. Since analyte inhibits antibody binding to the respective immobilized conjugate, increasing concentrations of analyte will reduce the phase decrease of the piezoelectric sensor (a general scheme showing the sensing interface and the signal transduction strategy is provided as supplementary information, Fig. S3). Carbaryl and TBZ standard solutions ranging from $10^{-3}$ to $2.0 \mathrm{x}$ $10^{3} \mu \mathrm{g} \mathrm{L}{ }^{-1}$ were prepared from $1 \mathrm{mM}$ stock solutions in dimetylformamide (stored at $20^{\circ} \mathrm{C}$ in dark vials) by serial dilutions in PBS (10 mM phosphate buffer solution, $0.9 \%$ $\mathrm{NaCl}, \mathrm{pH}$ 7.4). For carbaryl assays, depending on the crystal operational fundamental frequency, the standards were mixed with different fixed concentrations of LIB-CNH45 MAb in PBS: $2.5 \mu \mathrm{g} \mathrm{mL}^{-1}$ for $50 \mathrm{MHz}$ and $2.0 \mu \mathrm{g} \mathrm{mL}^{-1}$ for $100 \mathrm{MHz}$ resonators. For TBZ, $1.0 \mu \mathrm{g} \mathrm{mL}^{-1}$ of LIB-TN3C13 MAb was used in combination with $100 \mathrm{MHz}$ crystals. Analyte-antibody mixture solutions were incubated for $1 \mathrm{~h}$ at room temperature and then $250 \mu \mathrm{L}$ of this mixture were brought onto the sensor surface. Variations in phase response were monitored in real time as the binding between free antibody and the immobilized conjugate took place. Regeneration of the functionalized surfaces was accomplished with $0.1 \mathrm{M} \mathrm{HCl}$ to break the antibody-hapten conjugate association in carbaryl immunoassays and $0.1 \mathrm{M} \mathrm{NaOH}$ to break the antibody-conjugate interaction in TBZ assays.

All standards and samples were run at least in duplicate. Standard curves were obtained by plotting the phase decrease versus analyte concentration. The experimental points were fitted to the four-parameter logistic equation:

$$
y=y_{\mathrm{o}}+\left[\left(A-y_{\mathrm{o}}\right) / 1+\left(x / x_{\mathrm{o}}\right)^{B}\right]
$$

where $y$ is the HFF-QCM signal (variation of phase at the fixed fundamental frequency $\Delta \varphi), y_{\mathrm{o}}$ is the asymptotic minimum (background signal), $A$ is the asymptotic maximum (maximum signal in absence of analyte, $\mathrm{S}_{\mathrm{max}}$ ), $x$ is the analyte concentration, $x_{\mathrm{o}}$ is the analyte concentration giving $50 \%$ inhibition $\left(\mathrm{I}_{50}\right)$, and $B$ is the slope of the sigmoidal curve at the inflection point.

Mean standard curves were obtained by averaging several individual standard ones. These curves were normalized by expressing the phase decrease provided by each standard concentration as the percentage of the maximum response (maximum signal, $\left.\mathrm{S}_{\max }=100 \%\right)$ in the absence of analyte. 


\subsection{Immunoassay protocol}

To block non-specific MAb adsorption to the functionalized gold surface, prior to the first assay on every freshly immobilized crystal, blocking buffer (PBS containing $0.5 \%$ BSA) was pumped for $15 \mathrm{~min}$ at $20 \mu \mathrm{L} \mathrm{min}{ }^{-1}$. At this stage, only very low variations of phase ( $\Delta \varphi$ measured as voltage variation, $\Delta u \varphi \leq 10 \mathrm{mV})$ were obtained and signal baseline was easily recovered with working buffer (PBST: PBS containing $0.005 \%$ Tween 20$)$. The assay procedure was as follows:

(1) 4-5 min flow of working buffer (PBST) to stabilize the baseline signal.

(2) Sample injection $(250 \mu \mathrm{L})$ and 15 min flow of working buffer.

(3) Regeneration (4 min) with $0.1 \mathrm{M} \mathrm{HCl}$ (carbaryl) or $\mathrm{NaOH}$ (TBZ) followed by working buffer (1-2 min).

At steps (1) and (2) the flow rate was of $20 \mu \mathrm{L} \min ^{-1}$. For step (3) it was raised to 250 or $150 \mu \mathrm{L} \mathrm{min}^{-1}$ for carbaryl or TBZ assays, respectively. A complete assay cycle, including regeneration, takes around $25 \mathrm{~min}$. Once the last assay had finished, everyday crystals were washed with double distilled water, air-dried and stored at $4^{\circ} \mathrm{C}$.

\section{Results and discussion}

\section{1. $50 \mathrm{MHz} H F F-Q C M$ carbaryl immunosensor}

As for most immunosensors, operating with HFF QCMs requires the assessment of the optimal concentrations of immunoreagents involved in the antigen-antibody binding. As known, low molecular weight compounds as carbaryl and thiabendazole should be measured by means of competitive immunoassays (Abad et al., 2001; March et al., 2009). Moreover, indirect competitive immunoassays, in combination with covalent protein immobilization via SAM, provide the highest sensor sensitivity, regeneration capability and reproducibility. In the present work, optimization of immunoreagent concentrations was performed for carbaryl as a model analyte.

\subsubsection{Immunoassay optimization: Selection of the optimal assay conjugate concentration and electrode diameter}

The pair [BSA-CNH conjugate] - [LIB CNH45 monoclonal antibody] was previously selected as the optimal carbaryl antigen-antibody pair for QCM immunoassays (March et al., 2009). First, 5 and $10 \mathrm{mg} \mathrm{mL}^{-1}$ of BSA-CNH were 
immobilized onto $50 \mathrm{MHz}$ QCM SAM-activated electrodes with electrode diameter ranging from 1.0 to $2.0 \mathrm{~mm}$. The concentrations of thiolated compounds were roughly proportional to their respective conjugate concentration and low enough as to produce well organized and compacted monolayers (Chaki and Vijayamohanan, 2002). LIBCNH45 MAb concentration was fixed at $15.6 \mu \mathrm{g} \mathrm{mL}^{-1}$ and, once delivered to the flow cell, changes in phase response in $\mathrm{mV}(\Delta u \varphi)$ were measured as antigen (BSA-CNH) antibody (LIB-CNH45) interaction took place.

For 5 and $10 \mathrm{mg} \mathrm{mL}^{-1}$ of immobilized conjugate, electrode surfaces of 2.0 and $1.5 \mathrm{~mm}$ diameter gave the highest and very similar signals, whereas $1 \mathrm{~mm}$ electrode diameter provided poorer phase shifts. The longest electrode diameter $(2.0 \mathrm{~mm})$ also produced the highest baseline drifts and signal noise. Under the same conditions, resonators with $1.5 \mathrm{~mm}$ electrode diameter appeared to be the most efficient since they gave the highest signal variation to electrode diameter ratio (average $\Delta u \varphi / \varnothing$ of 1568 for $1.5 \mathrm{~mm} v s 825$ for $2.0 \mathrm{~mm}$, see supplementary data, table S1).

In relation to the thiolated compounds used for SAM formation, signal variations were fairly similar for both MHDA and TA. MHDA has the longest aliphatic chain, a quality that empowers the assembly of well-ordered monolayers and therefore enhances the efficiency of the covalent binding. Subsequently, lower conjugate concentrations ranging from 0.001 to $1.0 \mathrm{mg} \mathrm{mL}^{-1}$ were immobilized only on $1.5 \mathrm{~mm}$ diameter electrode sensors, employing MHDA as the thiol compound for SAM generation. For BSA-CNH conjugate concentrations in the $0.01-1.0 \mathrm{mg} \mathrm{mL}^{-1}$ range, the obtained phase shifts were the highest and very similar among them (supplementary data, table S1). BSA-CNH conjugate immobilized at $0.01 \mathrm{mg} \mathrm{mL}^{-1}$ produced the highest signal variation with the lowest immunoreagent expense.

\subsubsection{Immunoassay optimization: Selection of the optimal monoclonal antibody concentration}

Several concentrations of MAb (LIB-CNH45) ranging from 1.0 to $50.0 \mu \mathrm{g} \mathrm{mL}^{-1}$ were assayed in combination with 1,10 and $100 \mu \mathrm{g} \mathrm{mL}^{-1}$ of BSA-CNH conjugate immobilized on $50 \mathrm{MHz}$ sensor chips with $1.5 \mathrm{~mm}$ electrode diameter. Obtained results are displayed in Fig. 1 (triangles). For every conjugate concentration, the HFF-QCM signal was plotted against its corresponding MAb dose. As expected, higher binding corresponded to increasing both MAb and conjugate concentrations. For $50 \mathrm{MHz}$ sensors, $20 \mu \mathrm{g} \mathrm{mL}^{-1}$ of antibody provided signals near saturation. Furthermore, to fulfill 
the requirements for competitive immunoassays, the selected MAb concentration should not exceed $50 \%$ of the maximum measured signal. Subsequently, the optimum LIBCNH45 MAb concentration should be $\leq 5 \mu \mathrm{g} \mathrm{mL}^{-1}$. At any antibody concentration the binding signal obtained for $10 \mu \mathrm{g} \mathrm{mL}^{-1}$ conjugate was almost identical to that of $100 \mu \mathrm{g}$ $\mathrm{mL}^{-1}$, whereas it drastically dropped at the lowest conjugate concentration $\left(1 \mu \mathrm{g} \mathrm{mL}^{-1}\right)$.

In summary, for $50 \mathrm{MHz}$ sensor chips these results confirmed the selection of 10 $\mu \mathrm{g} \mathrm{mL} L^{-1} \mathrm{BSA}-\mathrm{CNH}$ as the optimal conjugate concentration for immobilization. As regards to antibody optimal concentration, $2.5 \mu \mathrm{g} \mathrm{mL} \mathrm{m}^{-1}$ of MAb provided optimal signals with the lowest immunoreagent consumption.

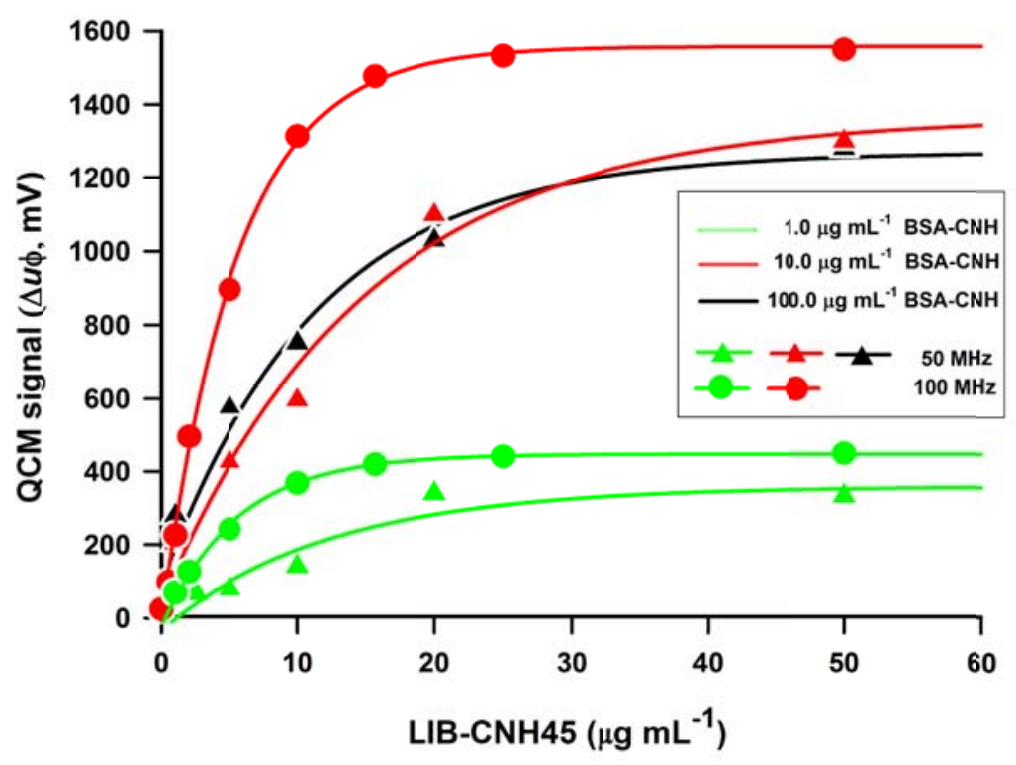

Fig. 1. Optimization of the HFF-QCM carbaryl immunoassay. Signal variation obtained with $50 \mathrm{MHz}$ (triangles) and $100 \mathrm{MHz}$ (circles) QCM resonators as a function of LIB-CNH45 MAb concentration for three BSA-CNH conjugate concentrations: $1 \mu \mathrm{g} \mathrm{mL}^{-1}$ (green), $10 \mu \mathrm{g} \mathrm{mL}^{-1}$ (red), and $100 \mu \mathrm{g} \mathrm{mL}^{-1}$ (black).

\subsection{3. $50 \mathrm{MHz} H F F-Q C M$ standard curves: Assay sensitivity}

Fig. 2 shows a representative real-screen record of signals obtained for different carbaryl concentrations subjected to competitive immunoassays in the same experiment. The lower record represents the phase shifts produced when conjugate-MAb binding occurred. The real-time sensograms include interaction (binding events) and regeneration steps. The interaction took approximately $15 \mathrm{~min}$. As expected for competitive assays, phase shifts decreased when carbaryl concentrations increased. MAb solution was injected every two or three assays to control the constant and slight baseline dropping throughout the assays. 
Two more additional parameters were measured and recorded simultaneously: amplitude (middle record) and temperature (upper record). As shown, temperature was nearly constant $\left(25.00 \pm 0.05^{\circ} \mathrm{C}\right)$ through the whole experiment. Regarding amplitude, it also changed with binding events. At low carbaryl concentrations, phase shifts were higher than amplitude ones. However, as analyte concentration increased, the observed amplitude variations were higher than those of phase.

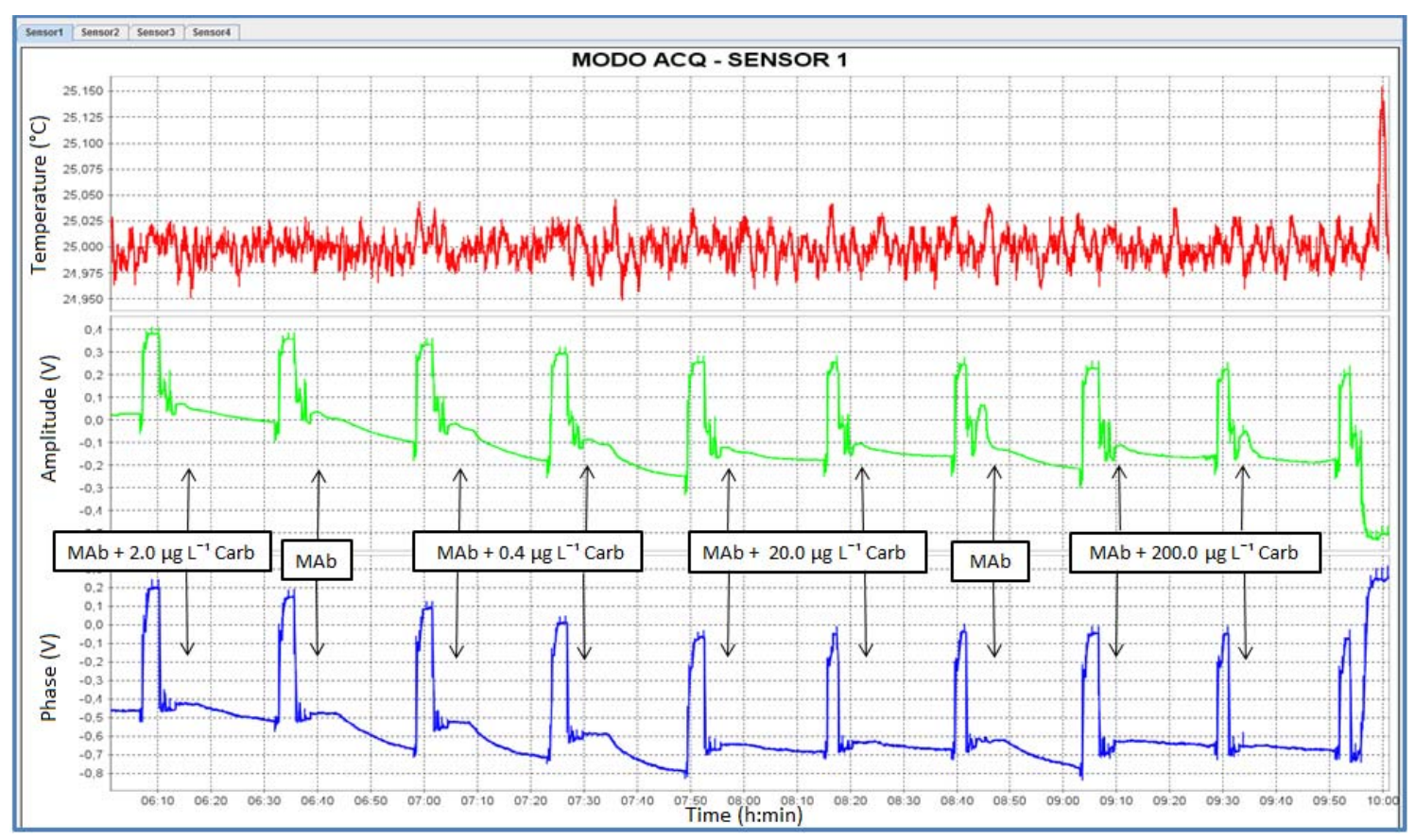

Fig. 2. HFF-QCM immunosensor response to analyte concentration. Upper, middle and lower record: real-time monitoring of temperature, amplitude and phase, respectively. The three records correspond to consecutive carbaryl immunoassays performed with a constant, optimized LIB-CNH45 MAb concentration. Arrows indicate sample injection. Regeneration steps appear as high peaks.

Biochemical processes that take place on the sensor surface (adsorption, binding, etc.) cause changes in both phase $(\Delta \varphi)$ and amplitude $(\Delta A): \Delta \varphi$ corresponds to the amount of bound mass and $\Delta A$ to conformation of the bound biomolecules or viscoelastic properties at the sensor-liquid interface (Mitsakakis and Gizeli, 2011).The amplitude change to phase change ratio $(\Delta A / \Delta \varphi)$ provides an insight on viscosity changes occurring at the sensor-liquid interface. Some researchers concluded that experimental high $\Delta A / \Delta \varphi$ ratios are reliable indicators of viscous interactions, whereas small ratios indicate a rigid layer (Tsortos et al., 2008), as desirable for reliable microgravimetric measurements (Kanazawa and Gordon, 1985). Taking into account that carbaryl assays in the immunosensor are competitive, the larger changes in phase than in amplitude obtained for low carbaryl concentrations and the smaller changes in 
phase than in amplitude produced by high carbaryl concentrations, strongly suggest that $\Delta \varphi$ mainly accounts for mass changes on the sensor surface. For the purpose of this work, measuring phase changes were indeed enough to quantify the antibody-antigen interactions. Thus, the immunosensor was working in the microgravimetric mode.

Hence, phase shifts were used to generate carbaryl immunosensor standard curves. They were obtained by averaging three individual ones performed with at least two assays per carbaryl concentration. Voltage phase shifts $(\Delta u \varphi)$ were normalized by expressing them as: $100 \times \Delta u \varphi / \Delta u \varphi_{0}$, where $\Delta u \varphi_{0}$ is the voltage phase decrease at zero analyte concentration (maximum signal). Experimental points were fitted to the mathematical logistic function according to equation (3).

As depicted in Fig. 3 (triangles, black line), the carbaryl standard curve for the $50 \mathrm{MHz}$ QCM immunosensor showed the typical sigmoidal shape of competitive assays, where signal decreases as analyte concentration increases. $\mathrm{I}_{50}$ value, which is an estimate of assay sensitivity, was $1.95 \mu \mathrm{g} \mathrm{L}^{-1}$; the limit of detection (LOD: $\mathrm{I}_{90}$ value) was $0.23 \mu \mathrm{g} \mathrm{L}^{-1}$, and the working range (WR: $\mathrm{I}_{80-20}$ range) was $0.50-7.20 \mu \mathrm{g} \mathrm{L}^{-1}$.

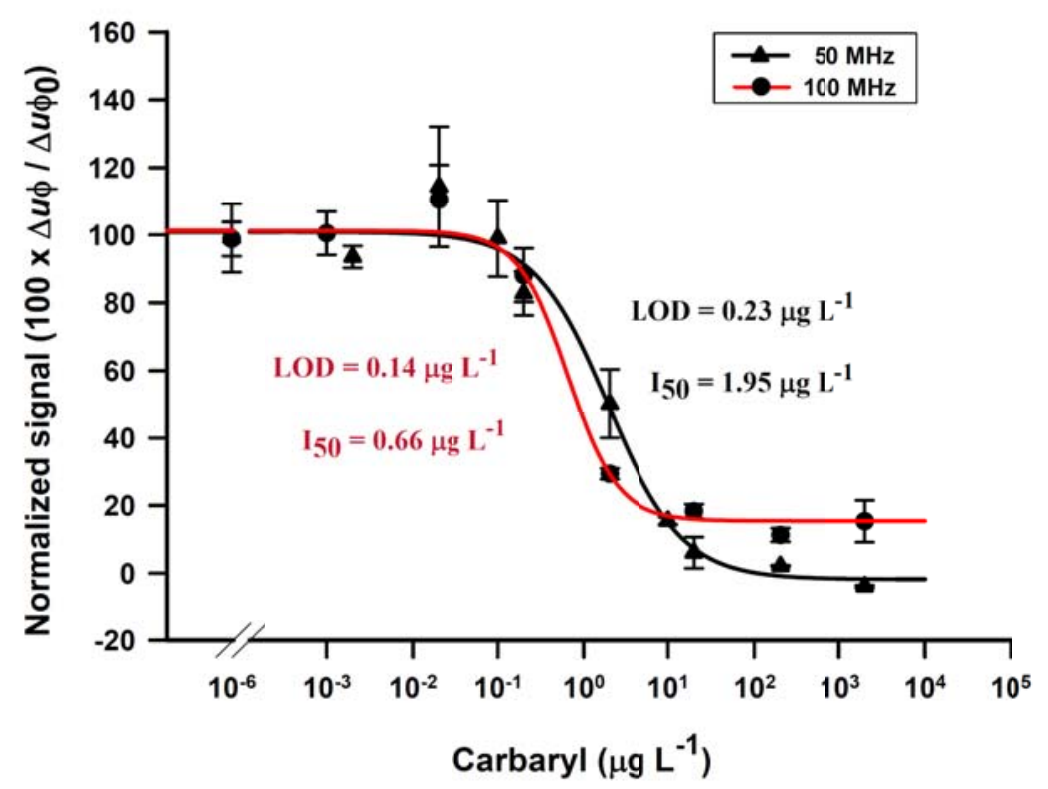

Fig. 3. HFF-QCM carbaryl standard calibration curves performed with $50 \mathrm{MHz}$ (triangles) and $100 \mathrm{MHz}$ (circles) resonators under optimized conditions.

\section{2. $100 \mathrm{MHz} H F F-Q C M$ immunosensors}

\subsubsection{Carbaryl immunoassay optimization: Selection of the immunoreagent} concentrations 
For $100 \mathrm{MHz}$ QCM immunosensors, carbaryl was also used as a model analyte. Antigen-antibody dose-response curves were performed for only two BSA-CNH conjugate concentrations: 1 and $10 \mu \mathrm{g} \mathrm{mL}^{-1}$. In this case saturation was again reached close to $20 \mu \mathrm{g} \mathrm{mL}^{-1}$ (Fig. 1, circles). Slopes were higher than those of $50 \mathrm{MHz}$ at the same conjugate doses. Not surprisingly, the lowest signals were obtained with the lowest conjugate concentration $\left(1 \mu \mathrm{g} \mathrm{mL}^{-1}\right)$. The binding signal for $10 \mu \mathrm{g} \mathrm{mL}^{-1}$ conjugate was high enough as not to consider higher conjugate concentrations worthwhile to be assayed. These results are in agreement with those of $50 \mathrm{MHz}$ and confirm the selection of $10 \mu \mathrm{g} \mathrm{mL}^{-1}$ as the optimal concentration for BSA-CNH conjugate immobilization, for either 50 or $100 \mathrm{MHz}$ resonators. The higher responses obtained with $100 \mathrm{MHz}$ enabled lowering the LIB-CNH45 MAb concentration for the competitive assay down to $2.0 \mu \mathrm{g} \mathrm{mL}^{-1}$.

In previous works with conventional $9 \mathrm{MHz}$ QCM sensors, the MAb doseresponse curve at $10 \mathrm{mg} \mathrm{mL}^{-1}$ assay conjugate reached saturation at around $200 \mu \mathrm{g} \mathrm{mL}^{-1}$ MAb (March et al. 2009). For 50 and $100 \mathrm{MHz}$ resonators, the saturating concentration was roughly one order of magnitude lower than that of $9 \mathrm{MHz}$ sensors, which certainly pointed towards an improvement of at least one order of magnitude in sensor sensitivity. This improvement allowed us to significantly reduce not only the MAb but also the conjugate concentration: they were around one and three orders of magnitude, respectively, lower than those employed for the $9 \mathrm{MHz}$ carbaryl immunosensor.

\subsubsection{Carbaryl standard curves: Electrode diameter selection and assay sensitivity}

For $100 \mathrm{MHz}$ QCM immunosensors, preliminary experiments were performed with the same electrode diameter previously selected for the $50 \mathrm{MHz}$ immunosensor: $1.5 \mathrm{~mm}$. But, when the highest carbaryl concentrations were assayed, phase variations were too large and yielded poor calibration curves with unacceptable asymptotic minimum over $30 \%$ of the maximum assay signal (data not shown). Other drawbacks found were baseline drifts and some unsteady sensor response. This unsuitable behavior seemed to decrease when lower electrode surfaces (1.0 and $0.5 \mathrm{~mm}$ diameter) were used. The performance of $0.5 \mathrm{~mm}$ diameter sensors was not adequate because signal decayed to undesirable levels. In contrast, immunoassays performed on functionalized $1.0 \mathrm{~mm}$ diameter electrodes provided standard curves with quite good sensitivity, similar to that of $1.5 \mathrm{~mm}$ but with an acceptable asymptotic minimum below $20 \%$ of the maximum signal. 
The unadvisable response provided by $1.5 \mathrm{~mm}$ in relation to that of $1.0 \mathrm{~mm}$ electrode diameter $100 \mathrm{MHz}$ resonators could be explained in terms of theoretical considerations on the crystal quality factor. In brief, HFF-QCM resonators have lower quality factors than conventional low frequency ones. In addition, this factor further decreases in usual liquid applications, such as biosensors, in comparison with "in air". That is, in liquid media, as evidenced from the conductance-frequency relationship, a dramatic increase in the width of the conductance peak as well as a decrease in the maximum conductance accompanies liquid loading (Lin et al., 1993). This fact can account for the overlapping between spurious signals and fundamental resonance peak. In order to minimize this effect in HFF-QCM resonators, additional studies pointed towards a reduction of the electrode diameter in consonance with the quartz thickness decrease (Kao et al., 2008). Ever since 1964, King Jr. postulated that the sensitivity of detection was inversely proportional to the square of the electrode diameter and the resonator thickness. Recently, Liang et al. (2013) reported that for HFF-QCM chips of about $73 \mathrm{MHz}$, quality factor increases as electrode diameter decreases for a given range. Thus, there is a need to find out a crystal design compromise in order to get the optimal electrode diameter to crystal thickness ratio for a given HF frequency. In our case, $1.0 \mathrm{~mm}$ seemed to be better than $1.5 \mathrm{~mm}$ electrode diameter for our $100 \mathrm{MHz}$ HFF-QCM immunosensor application.

Calibration curves were then obtained on $100 \mathrm{MHz}, 1.0 \mathrm{~mm}$ electrode diameter sensors, functionalized with $10 \mu \mathrm{g} \mathrm{mL}^{-1}$ of BSA-CNH conjugate and using $2.0 \mu \mathrm{g} \mathrm{mL}^{-1}$ of LIB-CNH45 MAb. As for that of $50 \mathrm{MHz}$, the $100 \mathrm{MHz}$ carbaryl standard curve depicted in Fig. 3 (circles, red line) is the mean of three individual ones. $\mathrm{I}_{50}$ value was $0.66 \mu \mathrm{g} \mathrm{L}^{-1}$, LOD was $0.14 \mu \mathrm{g} \mathrm{L}^{-1}$ and WR was $0.26-1.73 \mu \mathrm{g} \mathrm{L}^{-1}$.

From these analytical parameters, an effect on the sensitivity could be observed with $100 \mathrm{MHz}$ in relation to $50 \mathrm{MHz}$ QCM sensor chips: sensitivity parameters for 100 $\mathrm{MHz}$ chips were around twice smaller than those of $50 \mathrm{MHz}\left(0.66 \mu \mathrm{g} \mathrm{L}{ }^{-1} v s 1.95 \mu \mathrm{g} \mathrm{L}^{-1}\right.$ $\mathrm{I}_{50}$, and $0.14 \mu \mathrm{g} \mathrm{L}^{-1}$ vs $0.23 \mu \mathrm{g} \mathrm{L}^{-1} \mathrm{LOD}$, for 100 and $50 \mathrm{MHz}$, respectively). These results confirmed those obtained in antigen-antibody dose-response curves depicted in Fig. 1, in which slopes for $100 \mathrm{MHz}$ were higher than slopes for $50 \mathrm{MHz}$. Moreover, as expected, lowering the antibody concentration as allowed by $100 \mathrm{MHz}$ QCM sensors lead to improved analytical sensitivity parameters. 


\subsubsection{Thiabendazole HFF-QCM immunosensor}

To further explore the potential applications of HFF-QCM technology for sensitive pesticide determination, a TBZ immunosensor was also developed. To this purpose, experimental parameters like SAM composition, assay conjugate and MAb concentrations were fixed according to those reported for SPR (Estevez et al., 2012). Briefly, $1.0 \mu \mathrm{g} \mathrm{mL}^{-1}$ of LIB-TN3C13 MAb was used on sensors functionalized with 20 $\mu \mathrm{g} \mathrm{mL}^{-1}$ of BSA-TN3C conjugate. The resonator employed was $100 \mathrm{MHz}$ with $1.0 \mathrm{~mm}$ diameter active electrode, in accordance with those previously selected as optimal fundamental frequency and electrode size HFF-QCM sensor chip for carbaryl.

Fig. 4 represents the mean of two individual TBZ curves. Phase shifts obtained for TBZ standards in the $10^{-3}-5.0 \times 10^{2} \mu \mathrm{g} \mathrm{L}^{-1}$ range were very similar to those obtained for carbaryl. As regards to the functionalized surface regeneration, a strong basic solution $(0.1 \mathrm{M} \mathrm{NaOH})$ had to be employed for a shorter time period (1-2 min), in order to ensure the effective LIB-TN3C13 monoclonal antibody detachment from the sensor surface. The $\mathrm{I}_{50}$ value, LOD and working range were: $0.80,0.06$ and $0.16-3.68 \mu \mathrm{g} \mathrm{L}^{-1}$ respectively. These analytical parameters are roughly the same order of magnitude as those previously reported for TBZ in ELISA (Abad et al., 2001) and in the SPR immunosensor (Estevez et al., 2012).

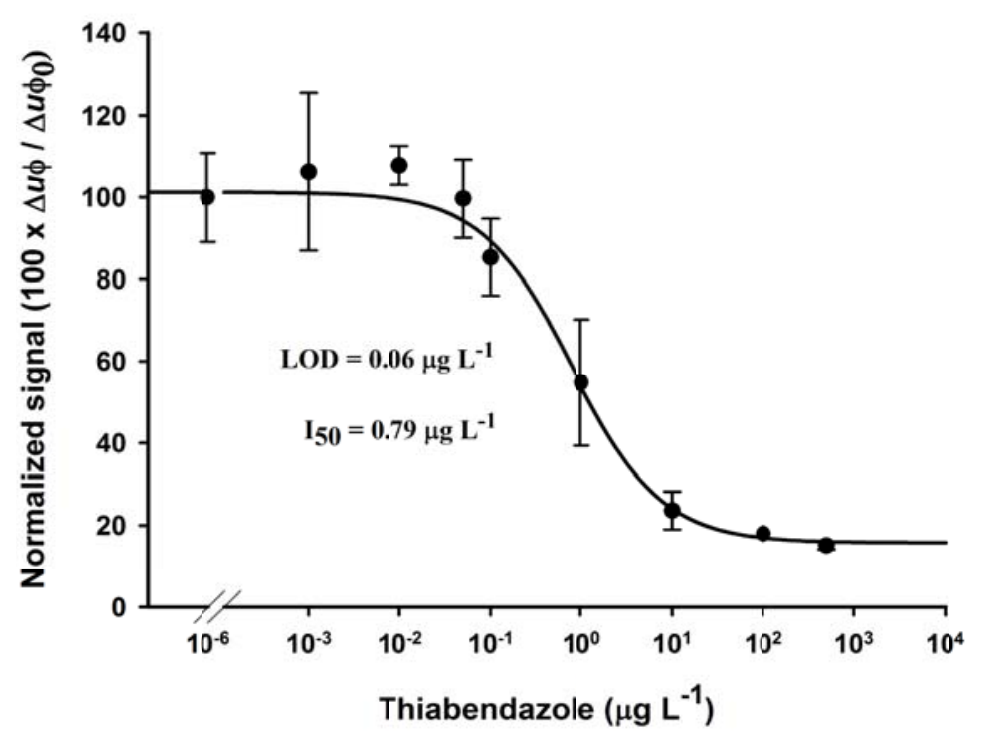

Fig. 4. Standard calibration curve for thiabendazole performed with $100 \mathrm{MHz}$ QC'M resonator, under optimized conditions.

\subsection{Comparison between pesticide immunosensors and ELISAs}

The parameters obtained from carbaryl standard curves with the $50 \mathrm{MHz}$ QCM sensor (Fig. 3, triangles, black line) entailed a 50-fold decrease in the LOD with respect 
to that for conventional $9 \mathrm{MHz}$ QCM crystals (March et al., 2009). Further LOD improvement (up to two orders of magnitude) was achieved by increasing twice the sensor fundamental frequency (100 MHz, Fig. 3, circles, red line). These results are summarized in Table 1, where the performance of different carbaryl immunosensors, under roughly the same biochemical conditions, is compared. As shown, for the three analytical parameters studied ( $\mathrm{I}_{50}$ value, $\mathrm{LOD}$ and $\left.\mathrm{WR}\right), \mathrm{QCM}$ immunosensors operating at high fundamental frequency (50 or $100 \mathrm{MHz}$ ) gave lower values than SPR. The limit of detection and $\mathrm{I}_{50}$ value achieved with $100 \mathrm{MHz}$ resonators were one order of magnitude and five-fold lower than their respective values reported for SPR (Mauriz et al., 2006). Nevertheless, the three immunosensing methodologies (conventional QCM, SPR and HFF QCM) still yielded higher $\mathrm{I}_{50}$ values (indicating a lower sensitivity) than the previously reported optimized ELISA (Abad and Montoya, 1997). This ELISA was in fact around one order of magnitude more sensitive than the best QCM immunosensor (100 MHz). However, the immunoreagents employed in the optimized ELISA could not be used in the high frequency QCM because of the immunosensor regeneration requirements. Anyway, if the comparison was established between the $100 \mathrm{MHz}$ QCM immunosensor and the ELISA performed under the same biochemical conditions (Abad et al., 1997), HFF QCM reached the same analytical performance. As regards to the working ranges for high frequency QCM, they were wider than those reported for SPR and conventional QCM and closer to ELISA working range.

Table 1

Analytical performance comparison between carbaryl immunosensors (QCM and SPR) and ELISA.

\begin{tabular}{|c|c|c|c|c|c|c|c|}
\hline \multirow{2}{*}{$\begin{array}{c}\text { Analytical } \\
\text { parameters } \\
\left(\mu \mathrm{g} \mathrm{L}^{-1}\right)\end{array}$} & \multicolumn{2}{|c|}{ ELISA } & \multirow{2}{*}{$\mathrm{SPR}^{3}$} & \multicolumn{4}{|c|}{ QCM: Fundamental Resonance Frequency (MHz) } \\
\hline & Standard $^{1}$ & Optimized $^{2}$ & & $9^{4, \mathrm{a}}$ & $10^{5, \mathrm{~b}}$ & $50^{\mathrm{b}}$ & $100^{\mathrm{b}}$ \\
\hline LOD & 0.13 & 0.01 & 1.41 & 13.30 & 4.00 & 0.23 & 0.14 \\
\hline $\mathrm{I}_{50}$ & 0.72 & 0.06 & 3.12 & 30.34 & 16.70 & 1.95 & 0.66 \\
\hline WR & $0.23-2.36$ & $0.02-0.18$ & $1.91-5.75$ & $18.30-50.30$ & $7.00-35.00$ & $0.50-7.20$ & $0.26-1.72$ \\
\hline & \multicolumn{7}{|c|}{$\begin{array}{l}{ }^{1} \text { Standard ELISA performed with the same immu } \\
\text { al., 1997) } \\
{ }^{2} \text { Abad and Montoya, } 1997 \\
{ }^{3} \text { Mauriz et al., } 2006 \\
{ }^{4} \text { March et al., } 2009 \\
{ }^{5} \text { Montagut et al., } 2011 \\
{ }^{a} \text { Frequency shift measurements } \\
{ }^{b} \text { Phase shift measurements at a fixed frequency. }\end{array}$} \\
\hline
\end{tabular}


For the thiabendazole fungicide, LOD was about 3 times higher than in ELISA and 2 times lower than in SPR performed under the same biochemical conditions (Table 2). Moreover, as expected from the lower slope of the HFF-QCM immunosensor standard curve, its working range was two-fold widened with respect to that of SPR (Estevez et al., 20012), thus reaching the same width as the reference ELISA (Abad et al., 2001).

Table 2

Analytical performance comparison between thiabendazole immunosensors (HFF-QCM and SPR) and ELISA.

\begin{tabular}{cccc}
\hline $\begin{array}{c}\text { Analytical parameters } \\
\left(\mu \mathrm{g} \mathrm{L}^{-1}\right)\end{array}$ & ELISA $^{1}$ & $\mathrm{SPR}^{2}$ & $\begin{array}{c}100 \mathrm{MHz} \\
\text { HFF-QCM }\end{array}$ \\
\hline LOD & 0.02 & 0.13 & 0.06 \\
$\mathrm{I}_{50}$ & 0.2 & 0.64 & 0.80 \\
WR & $0.05-0.90$ & $0.23-1.88$ & $0.16-3.68$ \\
\hline${ }^{1}$ Abad et al, 2001 & & &
\end{tabular}

\section{Conclusions}

Despite the widespread use of QCM technology, some challenges such as the improvement of the sensitivity and the limit of detection of QCM biosensors still remained unsolved. With the help of a new electronic sensor characterization approach, a sensitive HFF-QCM immunosensor has successfully been developed and tested for its use in pesticide determinations. This novel technology is based on the phase/mass relationship and allows a sensitive mass sensing by means of phase change measurements. Moreover, it also admits not only phase but also amplitude measurements, which could in turn allow the analysis of mass and viscosity changes during bio-interactions on the basis of the acoustic ratio (Dewilde et al., 2013).

Carbaryl insecticide chosen as a model analyte allowed us to compare the analytical performance of several immunosensors with respect to that of ELISA taken as the reference immunoassay. Analytical parameters ( $\mathrm{I}_{50}$ value, $\mathrm{LOD}$ and working range) of the $100 \mathrm{MHz}$ HFF-QCM carbaryl immunosensor indicated enhanced sensitivity as regards to that of either QCM or SPR immunosensors. The highest improvement was attained for LOD: around 10 and 100-fold improvement with respect to those of SPR 
and conventional QCM, respectively. Hence, sensitivity parameters approached the previously reported for the optimized ELISA. These features proved that lower immunoreagent consumption is needed and higher analytical sensitivity is achieved when signal is empowered. With regards to the standard curve for TBZ fungicide immunosensor, sensitivity parameters were the same order of magnitude as those of SPR immunosensor and ELISA performed under the same conditions.

To the best of our knowledge, this is the first high frequency QCM immunosensor successfully developed for demanding analytical applications, such as pesticide detection. Despite the high sensitivity and versatility of the developed HFFQCM platform, further work is still required. The influence of electrode diameter/crystal thickness ratio on the signal, as well as the relationship between amplitude/phase and viscoelastic phenomena occurring at the sensor-liquid interface, should be dealt with promptly. Experiments aimed to increase sensitivity by improving immobilization methods, and to elucidate the contribution of the electrode diameter on signal quality, are now in progress.

\section{Acknowledgments}

We would like to acknowledge Federico Martin and Juan Antonio Buitrago for their excellent technical assistance. This work was supported by European Regional Development Fund (ERDF) and the Spanish Ministry of Economy and Competitiveness (DETECTA IPT-2012-0154-300000 project).

\section{References}

Abad, A., Manclús, J.J., Moreno, M.J., Montoya, A. 2001. J. AOAC Int. 84, 156-161. Abad, A., Montoya, A., 1997. J. Agric. Food Chem. 45, 1495-1501.

Abad, A., Primo, J., Montoya, A., 1997. J. Agric. Food Chem. 45, 1486-1494.

Chaki, N.K., Vijayamohanan, K., 2002. Biosens. Bioelectron. 17, 1-12.

Dewilde, A.H., Wang, G., Zhang, J., Marx, K.A., Therrien, J.M., 2013. Anal. Biochem. $439,50-61$.

Estevez, M.C., Belenguer, J., Gomez-Montes, S., Miralles, J., Escuela, A.M., Montoya, A., Lechuga, L.M., 2012. Analyst 137, 5659-5665. 
Kanazawa, K.K., Gordon, J.G., 1985. Anal. Chim. Acta 175, 99-105.

Kao, P., Patwardhan,.A., Allara, D. Tadigadapa, S., 2008. Anal. Chem. 80, 5930-5936.

King, Jr., W.H., 1964. Anal. Chem. 36, 1735-1739.

Liang, J., Huang, J., Zhang, T., Zhang, J., Xuefeng Li, Ueda T., 2013. Sensors 13, 12140-12148.

Lin, Z., Yip, C.M., Joseph, I.S., Ward, M.D., 1993. Anal. Chem. 11. 1546-1551.

March, C., Manclús, J.J., Jiménez, Y., Arnau, A., Montoya, A., 2009. Talanta 78, 827833.

Marx, K.A. 2003. Biomacromolecules 4, 1099-1120.

Marx, K.A., 2007. The quartz crystal microbalance and the electrochemical QCM : applications to studies of thin polymer films, electron transfer systems, biological macromolecules, biosensors, and cells, in : Steinem, C., Janshoff, A., (Eds), Piezoelectric Sensors. Springer, Berlin, pp. 371-429.

Mauriz, E., Calle, A., Abad, A., Hildebrant, A., Barceló, D., Montoya, A., Lechuga, L.M., 2006. Biosens. Bioelectron. 21, 2129-2136.

Michalzik, M., Wendler, J., Rabe, J., Büttgenbach, S., Bilitewski, U., 2005. Sensor. Actuat. B 105, 508-515.

Mitsakakis, K., Gizeli, E., 2011. Anal. Chim. Acta 699, 1-5.

Montagut, Y.J., García, J.V., Jiménez, Y., March, C., Montoya, A., Arnau, A., 2011. Sensors 11, 4702-4720.

Sagmeister, B.P., Graz, I.M., Schwödiauer, R., Gruber, H., Bauer, S., 2009. Biosens. Bioelectron. 24, 2643-2648.

Sauerbrey, G., 1959. Z. Phys. 155, 206-222.

Sota, H., Yoshimine, H., Whittier, R.F., Gotoh, M., Shiohara, Y., Hasegawa, Y., Okahata, Y., 2002. Anal. Chem. 74, 3592-3598.

Speight, R., Cooper, M.A., 2012. J. Mol. Recognit. 25, 451-473.

Stehrer, B.P., Schwödiauer, R., Bauer, S., Graz, I.M., Pollheimer, P.D., Gruber, H., 2010. Procedia Engineering 5, 835-837.

Tsortos, A., Papadakis, G., Mitsakakis, K., Melzak, K. A., Gizeli, E., 2008. Biophys. J. 94, 2706-2715.

Uttenthaler, E., Schráml, M., Mandel, J., Drost, S., 2001. Biosens. Bioelectron. 16, 735743.

Yoshimine, H., Kojima, T., Furusawa, H., Okahata, Y., 2011. Anal. Chem. 83, 87418747. 


\section{Figure captions}

Fig. 1. Optimization of the HFF-QCM carbaryl immunoassay. Signal variation obtained with $50 \mathrm{MHz}$ (triangles) and $100 \mathrm{MHz}$ (circles) QCM resonators as a function of LIBCNH45 MAb concentration for three BSA-CNH conjugate concentrations: $1 \mu \mathrm{g} \mathrm{mL}^{-1}$ (green), $10 \mu \mathrm{g} \mathrm{mL}^{-1}$ (red), and $100 \mu \mathrm{g} \mathrm{mL}^{-1}$ (black).

Fig. 2. HFF-QCM immunosensor response to analyte concentration. Upper, middle and lower record: real-time monitoring of temperature, amplitude and phase, respectively. The three records correspond to consecutive carbaryl immunoassays performed with a constant, optimized LIB-CNH45 MAb concentration. Arrows indicate sample injection. Regeneration steps appear as high peaks.

Fig. 3. HFF-QCM carbaryl standard calibration curves performed with $50 \mathrm{MHz}$ (triangles) and $100 \mathrm{MHz}$ (circles) resonators under optimized conditions.

Fig. 4. Standard calibration curve for thiabendazole performed with $100 \mathrm{MHz}$ QCM resonator, under optimized conditions. 